\title{
Combining $\mathrm{CT}$ and nuclear: a winning hybrid team
}

\author{
Philipp A. Kaufmann, MDand Oliver Gaemperli, MD
}

\section{See related article, pp. 201-211}

An important aspect in the management of patients with coronary artery disease (CAD) is consideration of the duality of morphology and function. ${ }^{1}$ Hence, a comprehensive assessment of CAD should include both information on coronary lesion morphology and myocardial perfusion, and ideally both should be obtained noninvasively to allow for optimal clinical decision making and reduce the risk inherent in invasive diagnostic procedures. While perfusion imaging has been feasible by noninvasive means using myocardial perfusion scintigraphy for more than 3 decades, the concept of noninvasive coronary angiography has only recently gained clinical acceptance by the introduction of multislice computed tomography (MSCT). The newer generation CT scanners have finally met temporal and spatial resolution requirements for clinical use and have provided the base for combination with complementary modalities into hybrid imaging.

Advances in image processing software and the advent of hybrid scanners have further paved the way for fusion of image data sets from different modalities. This technology avoids mental integration of functional and morphological images and facilitates a comprehensive interpretation of the combined data sets. Particularly in patients with CAD, by revealing the burden of anatomic coronary disease and its physiologic relevance, the hybrid approach can provide noninvasively unique information that may help to improve diagnosis and management. First pioneering attempts of image fusion were promising ${ }^{2}$ but their widespread use was precluded by issues of insufficient image resolution, the lack of dedicated fusion software, and often tedious and time-

From the Nuclear Cardiology Division, Cardiovascular center, University Hospital Zurich, Zurich, Switzerland.

Reprint requests: Philipp A. Kaufmann, MD, Nuclear Cardiology Division, Cardiovascular Center, University Hospital Zurich NUK C

42, Raemistrasse 100, 8091 Zurich, Switzerland; pak@usz.ch.

J Nucl Cardiol 2009;16:170-2.

$1071-3581 / \$ 34.00$

Copyright (C) 2009 by the American Society of Nuclear Cardiology. doi:10.1007/s12350-008-9048-7 consuming image processing. Today, hardware and software developments have significantly improved hybrid imaging. Particularly, the creation of dedicated fusion software packages that are now commercially available have helped to simplify image fusion ${ }^{3}$ and therefore facilitate its implementation in clinical practice. Thus, hybrid imaging is becoming more and more available in specialized centers; however, data on its clinical usefulness remain scarce.

In the present issue of the Journal of Nuclear Cardiology, Santana et $\mathrm{al}^{4}$ report on their clinical experience with hybrid imaging using 16- and 64-slice CT angiography (CTA) and myocardial perfusion imaging (MPI) with single photon emission computed tomography (SPECT) or positron emission tomography (PET) (the latter with 82-Rubidium) in a series of 50 patients. The use of fused images improved sensitivity and particularly specificity for diagnosing significant CAD compared to MPI alone and compared to the sideby-side analysis of MPI and CTA. The reference standard was invasive coronary angiography (ICA) and significant CAD was defined as at least one stenosis with $\geq 50 \%$ obstruction. Although these single point statistics failed to reach statistical significance the comparison of diagnostic performance using receiver operating characteristics (ROC) statistics yielded superiority of fusion imaging over the other methods. This diagnostic impact is further underscored by the fact that the fusion images led to a modification of the initial interpretation in $28 \%$ of the cases. Finally, the incremental value of hybrid imaging compared to side-by-side analysis seemed to be confined to patients with multivessel disease, where a $17 \%$ increase in sensitivity was noted.

With these important results Santana and coworkers ${ }^{5}$ build on existing experiences with hybrid imaging. The present observations emphasize the incremental diagnostic value of hybrid imaging in several clinical scenarios. On one hand, in low risk populations fusion imaging increases the confidence for ruling out CAD as reflected by the higher normalcy rate reported by Santana et al. This is particularly helpful in a "stepwise" CAD evaluation approach where the first study has yielded equivocal results and a second study is needed to rule out disease with final certainty. So far many of these patients end up being referred for ICA, while hybrid imaging now offers a substantially improved diagnostic confidence 
resulting in less equivocal findings which may allow to greatly reduce the number of patients unnecessarily exposed to the risks of invasive procedures. ${ }^{6}$ In patients with multivessel disease, on the other hand, the use of hybrid imaging may provide important comprehensive evaluation. In fact, patients with multivessel disease who usually are older than the population mentioned above, have more jeopardized myocardium and impaired left ventricular function as well as higher risk for cardiovascular events. The optimal appreciation of patients with multivessel disease is therefore very important to allow for timely and appropriate treatment. The use of hybrid imaging in these patients may not only improve diagnostic accuracy, but may also (by combination with different tracers) enable evaluation of hemodynamic relevance of coronary stenoses and assessment of viability in territories subtended by occluded arteries. Santana and colleagues demonstrate that particularly in patients with multivessel disease the value of fusion images lies beyond the simple addition of a further diagnostic study. This value originates from the accurate spatial association of perfusion defects and coronary stenoses, which allows assigning hemodynamic properties to even small vessels, and is underscored by the $28 \%$ rate of changes in diagnostic impression compared to the side-by-side interpretation. Ultimately, this completely noninvasive comprehensive approach to CAD allows to obtain important information prior to any invasive procedure, and to stratify patients to their appropriate treatment strategy.

An irritating problem when evaluating the diagnostic performance of comprehensive hybrid technologies is the lack of an appropriate gold standard. Although ICA is widely accepted as the gold standard for CAD assessment (and as such was used in the study by Santana et al) it remains purely morphologic and lacks information on hemodynamic severity of coronary lesions. Intrinsically, when comparing a method for assessing coronary morphology and myocardial perfusion (i.e., hybrid technique) with a purely morphologic gold standard (i.e., ICA) an imperfect match is unavoidable by nature of the tests used. Conversely, the finding that adding CTA to MPI improves accuracy is not surprising using the gold standard of ICA, as a morphologic test compares better to another morphologic test. For instance, if an intermediate stenosis (i.e., 50-70\% stenosis on ICA) does not cause ischemia, it turns into a false negative finding, although in fact this stenosis is most likely hemodynamically irrelevant. By adding CTA which then confirms an intermediate lesion this turns into a true positive finding. Paradoxically, this contradicts the wisdom that functional assessment is important and adds important diagnostic information to CAD evaluation. However, the incremental information is lost if the gold standard is ICA. It is impossible that adding MPI to CTA increases the clinical information if any information from MPI is overruled by CTA. This conceptual issue is the fundamental problem with diagnostic studies using hybrid technology and future studies should aim at using more complex or combined gold standards such as for example ICA with fractional flow reserve measurements or combination of ICA and nuclear techniques. Ultimately, the best metric against which these techniques will have to prove their validity will probably be outcome and very recent data support that combined noninvasive anatomical and functional assessment by use of SPECT with CTA may allow improved risk stratification. $^{7}$

In conclusion, hybrid imaging combining nuclear and $\mathrm{CT}$ has risen as a new and attractive cardiac imaging modality. The improvements in software and hardware have greatly simplified the use of this technique as evidenced by the fact that in the study by Santana et al different centers with different modalities (SPECT and PET) and different generations of CT scanners (16- and 64-slices) were successful. This will help to further promote its clinical application. However, many questions as to the real clinical usefulness of hybrid imaging still remain unanswered and further research should aim at assessing the impact of hybrid imaging on clinical decision making and outcome. In the near future, hybrid technology may play an important role in the assessment and management of patients with heart disease due to its comprehensive and noninvasive nature. Furthermore, the unique potential to enable identification of high-risk plaques using fusion of morphology and biology with molecularly targeted PET imaging is a furtheralthough probably not so near-future perspective that should encourage continuing research in this field.

\section{References}

1. Topol EJ, Nissen SE. Our preoccupation with coronary luminology. The dissociation between clinical and angiographic findings in ischemic heart disease. Circulation 1995;92:2333-42.

2. Schindler TH, Magosaki N, Jeserich M, et al. Fusion imaging: Combined visualization of 3D reconstructed coronary artery tree and 3D myocardial scintigraphic image in coronary artery disease. Int J Card Imaging 1999;15:357-68 (discussion 369-70).

3. Gaemperli O, Schepis T, Kalff V, et al. Validation of a new cardiac image fusion software for three-dimensional integration of myocardial perfusion SPECT and stand-alone 64-slice CT angiography. Eur J Nucl Med Mol Imaging 2007;34:1097-106.

4. Santana CA, Garcia EV, Faber TL, et al. Diagnostic performance of image fusion of myocardial perfusion imaging (MPI) and computed tomography coronary angiography. J Nucl Cardiol 2008. 
doi:10.1007/s12350-008-9019-z.

5. Faber TL, Santana CA, Garcia EV, et al. Three-dimensional fusion of coronary arteries with myocardial perfusion distributions: Clinical validation. J Nucl Med 2004;45:745-53.

6. Gaemperli O, Schepis T, Valenta I, et al. Cardiac image fusion from stand-alone SPECT and CT: Clinical experience. J Nucl Med 2007;48:696-703.
7. Van Werkhoven JM, Schuijf JD, Gaemperli O, et al. Prognostic value of multi-slice computed tomography and gated single photon emission computed tomography in patients with suspected coronary artery disease. J Am Coll Cardiol 2009; in press. 